\title{
CHRISTENSEN ZERO SETS AND MEASURABLE CONVEX FUNCTIONS ${ }^{1}$
}

\author{
PAL FISCHER AND ZBIGNIEW SLODKOWSKI
}

\begin{abstract}
A notion of measurability in abelian Polish groups related to Christensen's Haar zero set is studied. It is shown that a measurable homomorphism or a measurable Jensen convex function defined on a real linear Polish space is continuous.
\end{abstract}

1. Introduction. Fréchet, Sierpinski, Banach, Ostrowski, among others, were interested in the following question. Let $f: R \rightarrow R$ such that

$$
f(x+y)=f(x)+f(y) \text { for } x, y \in R
$$

or

$$
f\left(\frac{x+y}{2}\right) \leqslant \frac{f(x)+f(y)}{2} \quad \text { for } x, y \in R .
$$

The problem is to find conditions which ensure the continuity of $f$.

We believe that a similar program should be carried out when $f: X \rightarrow R$, where $X$ is an abelian Polish group or is a real linear Polish space and $f$ is measurable in some given sense.

The first problem was treated in locally compact groups; Banach and $\mathbf{L}$. Schwartz [4] among others extended it into topological vector spaces, and more recently Christensen considered this problem in abelian Polish groups. Christensen's result does not entirely cover the Lebesgue or Haar measurable cases. By enlarging the class of measurable sets we obtain a more general theorem which includes these cases. Furthermore, we treat equally the second problem in the same framework, and we believe that is the first time that this problem is studied in the infinite dimensional case.

2. Christensen measurability. In this section a notion of measurability in abelian Polish groups related to Christensen's Haar zero set is studied.

Let $(X,+)$ be an abelian Polish group. A set $A \subset X$ is a Haar zero set (see [2, p. 256]) if it is universally measurable and if there exists a probability measure $u$ on $X$ such that $\chi_{1} * u=0$. In the following a measure is a countably additive Borel measure extended to the universally measurable sets, and we denote by $\mathscr{N}(X)$ the set of all probability measures on $X$.

We present the following definitions.

Received by the editors February 23, 1979 and, in revised form, July 23, 1979.

AMS (MOS) subject classifications (1970). Primary 46A90; Secondary 28A10, 28A20.

Key words and phrases. Haar zero set, universally measurable set, convex functions.

${ }^{1}$ Work supported in part by the N. R. C. of Canada under grant A-8745. 
Definition 1. A set $P \subset X$ is a Christensen zero set if it is a subset of a Haar zero set.

Definition 2. A set $W \subset X$ is Christensen measurable if $W=A \cup P$, where $A$ is universally measurable and $P$ is a Christensen zero set.

The following proposition can be established.

Proposition 1. Let $(X,+)$ be an abelian Polish group. Then $\mathcal{C}$, the set of all Christensen measurable sets, is a o-algebra.

Proof. Let $W=A \cup P$ be a Christensen measurable set, where $A$ is universally measurable and $P$ is a Christensen zero set. Since any subset of a Christensen zero set is a Christensen zero set again, we can assume, without loss of generality that $A \cap P=\varnothing$. Then there exists a Haar zero set $P_{1} \supset P$ such that $P_{1} \cap A=\varnothing$. Hence $X \backslash W=\left[X \backslash\left(A \cup P_{1}\right)\right] \cup\left(P_{1} \backslash P\right)$. Since $X \backslash\left(A \cup P_{1}\right)$ is universally measurable and $P_{1} \backslash P \subset P_{1}$, we see that $X \backslash W \in \mathcal{C}$.

According to a theorem of Christensen [2, Theorem 1, p. 256] in an abelian Polish group a countable union of Haar zero sets is a Haar zero set. Using this theorem we can deduce easily that a countable union of Christensen measurable sets is a Christensen measurable set. Q.E.D.

From the proof of our previous proposition it follows immediately that the Christensen zero sets form a $\sigma$-ideal, which implies the following corollary.

Corollary 1. If $(X,+)$ is an abelian Polish group and if $X$ is a countable union of Christensen measurable sets, then at least one set from the countable union is not a Christensen zero set.

Since $(X, \mathcal{C})$ is a measurable space the measurability of a mapping in the Christensen sense can be defined in the usual way.

Definition 3. Let $X$ and $Y$ be abelian Polish groups. A mapping $f: X \rightarrow Y$ is said to be Christensen measurable if for each open set $O$ in $Y f^{-1}(O)$ is Christensen measurable in $X$.

We close this section by proving a lemma which will be useful later.

Lemma 1. Let $X$ be an abelian Polish group, let $A \subset X$ be universally measurable and let $U$ be an open subset of $R$. Then $A \times U$ is universally measurable in $X \times R$.

Proof. Let $m$ be an arbitrary Borel measure on $X \times R$, and denote by $m / X \times U$ the restriction of $m$ into $X \times U$. If we could show that $C=A \times U$ can be written as $B \cup Z$, where $B$ is a Borel set and $m / X \times U(Z)=0$, that would imply that $m(Z)=0$ since $Z \subset X \times U$ and furthermore we could deduce that if $C$ is universally measurable in $X \times U$, then it is universally measurable in $X \times R$.

Let $m \in \mathfrak{R}(X \times U)$. We define a set function $\tilde{m}$ by $\tilde{m}(B):=m(B \times U)$ for the Borel sets in $X$. Clearly $\tilde{m} \in \mathfrak{R}(X)$. We know that $A \subset X$ is universally measurable; therefore $A=B_{0} \cup Z$ where $B_{0}$ is a Borel set and $\tilde{m}^{*}(Z)=0\left(\tilde{m}^{*}\right.$ is the Carathéodory extension of $\tilde{m})$. In other words, there exists a Borel set $B_{1} \supset Z$ with $\tilde{m}\left(B_{1}\right)=0$. Therefore $A \times U=\left(B_{0} \times U\right) \cup(Z \times U)$, where we have $Z \times U$ $\subset B_{1} \times U, m\left(B_{1} \times U\right)=\tilde{m}\left(B_{1}\right)=0$. Q.E.D. 
3. Measurable homomorphisms. In this section we shall show that a Christensen measurable homomorphism is continuous.

Theorem 1. Let $(G,+)$ and $(H,+)$ be abelian Polish groups and let $f: G \rightarrow H$ be a Christensen measurable homomorphism; then $f$ is continuous.

Proof. Let $\overline{f(G)}=H_{0}$. Clearly $f(G)$ is a subgroup of $H$, and so is $H_{0}$. It is enough to show that $f: G \rightarrow H_{0}$ is continuous.

Let $U \in O_{H_{0}}$ (neighborhood of 0 in $H_{0}$ ); then there exists $V \in O_{H_{0}}$ such that $V-V \subset U$. Furthermore, $\overline{f(G)} \subset f(G)+V=H_{0}$, that is

$$
\bigcup_{g \in G}(f(g)+V)=H_{0} \text {. }
$$

Since a Polish space is a Lindelöf space, in (3) there exists a countable subcover, that is there is a sequence $\left\{g_{i}\right\}$ so that

$$
\bigcup_{i=1}^{\infty}\left(f\left(g_{i}\right)+V\right)=H_{0} \text {. }
$$

From (4) we obtain that

$$
\bigcup_{i=1}^{\infty} f^{-1}\left(f\left(g_{i}\right)+V\right)=G .
$$

Since $f^{-1}\left(f\left(g_{i}\right)+V\right)=\left\{x: f(x)=f\left(g_{i}\right)+V\right\}=\left\{x: \quad x-g_{i} \in f^{-1}(V)\right\}=g_{i}+$ $f^{-1}(V)$, it follows that $\cup_{i=1}^{\infty}\left(g_{i}+f^{-1}(V)\right)=G$. Using the fact that $G$ is not a Christensen zero set we can conclude that there exists an integer $n_{0}$ such that $g_{n_{0}}+f^{-1}(V)$ is not a Christensen zero set, or equivalently $f^{-1}(V)$ is not a Christensen zero set. Let $f^{-1}(V)=A \cup P$, where $A$ is universally measurable and $P$ is a Christensen zero set. Clearly $A$ is not a Haar zero set. By a theorem of Christensen [3, Theorem [7.3]] we can conclude that $A-A$ is a neighborhood of $0_{G}$, that is $f^{-1}(U)$ is a neighborhood of $0_{G}$. Since $U \in O_{H_{0}}$ was arbitrary, we can conclude that $f$ is continuous. Q.E.D.

It is worthwhile to mention the following three corollaries of the previous theorem.

Corollary 2. Let $X$ be a linear Polish space and let $f: X \rightarrow R$ be a Christensen measurable linear functional; then $f$ is continuous.

Corollary 3. Let $(X,+)$ be an abelian Polish group, and let $f: X \rightarrow R$ be a Christensen measurable homomorphism; then $f$ is continuous.

A special case of Corollary 3 is when $X=R$. In this case Christensen measurability is the same as Lebesgue measurability. Therefore Theorem 1 generalizes a theorem of Fréchet.

Corollary 4. Let $(X, \cdot)$ be an abelian Polish group, and let $f: X \rightarrow C$ be a Christensen measurable group character; then $f$ is continuous.

4. Measurable Jensen convex functions. In this section we shall consider Christensen measurable Jensen (or midpoint) convex functions. First we present some definitions. 
Definition 4. Let $X$ be a linear space. A set $V \subset X$ is said to be midpoint convex if $v_{1}, v_{2} \in V$ implies that $\left(v_{1}+v_{2}\right) / 2 \in V$.

In the rest of this paper we shall denote by $Q_{2}$ the set of all $\rho$ 's such that $\rho=n / 2^{m}$ for some integers $n$ and $m$.

Definition 5. Let $X$ be a linear space and let $V \subset X$. An $x_{0} \in V$ is said to be a $Q_{2}$-internal point of $V$ if for every $x \in X$ there exists an $\varepsilon(x)>0$ such that $x_{0}+\rho x \in V$ for $\rho \in Q_{2} \cap(-\varepsilon, \varepsilon)$.

We omit the easy proof of the following lemma.

Lemma 2. Let $X$ be a linear Polish space and let $\psi$ be a continuous affine one-to-one mapping of $X$ onto $X$; then $\psi$ maps a Christensen zero set into a Christensen zero set.

Now we can prove the main result of this section.

Theorem 2. Let $X$ be a real linear Polish space, let $f: X \rightarrow R$ be a Christensen measurable and Jensen convex mapping, i.e. $f((x+y) / 2) \leqslant(f(x)+f(y)) / 2$ for $x, y \in X$. Then $f$ is a continuous, convex mapping.

Proof. Let $W=\{(x, t) \in X \times R: f(x) \leqslant t\}$. To facilitate the description of the proof we shall consider some lemmas.

LEMMA 3. $W$ is a midpoint convex set.

We omit the obvious proof of this lemma.

Lemma 4. Let $\left(x_{0}, t_{0}\right) \in W$. Then $\left(x_{0}, t_{0}\right)$ is a $Q_{2}$-internal point of $W$ if and only if $f\left(x_{0}\right)<t_{0}$. $W$.

Proof. It is evident that if $f\left(x_{0}\right)=t_{0}$, then $\left(x_{0}, t_{0}\right)$ is not a $Q_{2}$-internal point of

Conversely, assume that $f\left(x_{0}\right)<t_{0}$. Let $(x, t)$ be an arbitrary point in $X \times R$. Let us consider the following four points: $A=\left(x_{0}, t_{0}\right), B=\left(x_{0}, f\left(x_{0}\right)\right), C=\left(x_{0}+x, t_{0}\right.$ $+t)$ and $D=\left(x_{0}+x, f\left(x_{0}+x\right)\right)$. Clearly these four points are coplanar. We shall distinguish three cases depending on whether $t_{0}+t<f\left(x_{0}+x\right), t_{0}+t=$ $f\left(x_{0}+x\right)$ or $t_{0}+t>f\left(x_{0}+x\right)$.

If $t_{0}+t<f\left(x_{0}+x\right)$, then the line going through the points $A$ and $C$ intersects the line going through the points $B$ and $D$ at a point $E$, which is between $A$ and $C$. The line segment $A E$ is above the line segment $B E$. Therefore all points in the line segment $A E$ which have the form $\left(x_{0}, t_{0}\right)+\eta(x, t)$ and $\eta \in Q_{2}$ belong to $W$. Cases 2 and 3 can be handled similarly. The fact that $(x, t)$ was chosen arbitrarily establishes this lemma.

Lemma 5. Let $X$ be a real linear Polish space, and let $V \subset X$ be a Christensen measurable and midpoint convex set; then the $Q_{2}$-internal points of $V$ form an open set.

Proof. Assume that $x_{0}$ is a $Q_{2}$-internal point of $V$, and consider the set $U=\left(V-x_{0}\right) \cap\left(-V+x_{0}\right) . U$ is symmetric with respect to the origin, Christensen measurable, midpoint convex and the origin is a $Q_{2}$-internal point of $U$. Hence 
$\cup_{n=1}^{\infty}\left(2^{n} U\right)=X$. We deduce from Corollary 1 and Lemma 2 that $U$ is not a Christensen zero set. Thus by repeating an argument from the proof of Theorem 1, we see that $U-U=2 U$ is a neighborhood of 0 . Hence $U$ is a neighborhood of 0 and $x_{0}+U \subset V$, which proves this lemma.

Lemma 6. Let $X$ be a linear Polish space, and let $f: X \rightarrow R$ be Christensen measurable. Then $W=\{(x, t) \in X \times R: f(x)<t\}$ is Christensen measurable.

Proof. Let $F(x, t)=(f(x), t): X \times R \rightarrow R^{2}$. We shall show first that $F$ is Christensen measurable. Clearly, it is enough to show that $F^{-1}\left(U_{1} \times U_{2}\right)$ is Christensen measurable, where $U_{1}, U_{2}$ are open sets in $R$. Let us remark that $F^{-1}\left(U_{1} \times U_{2}\right)=f^{-1}\left(U_{1}\right) \times U_{2}=(A \cup P) \times U_{2}=\left(A \times U_{2}\right) \cup\left(P \times U_{2}\right)$, where $A$ is universally measurable and $P$ is a Christensen zero set. By Lemma $1, A \times U_{2}$ is universally measurable in $X \times R$. Let $H$ be a Haar zero set in $X$ such that $H \supset P$. As before $H \times U_{2}$ is universally measurable in $X \times R$. We have to show that $H \times U_{2}$ is a Haar zero set. Since $H$ is a Haar zero set in $X$ there exists $\mu \in \mathfrak{N}(X)$ so that $\chi_{H} * \mu=0$. Now let $\mu_{1}=\mu \times \delta_{0}\left(\delta_{0}\right.$ is the Dirac measure on $R$ with support at 0$)$. Clearly $\mu_{1} \in \mathfrak{N}(X \times R)$ and if $(x, t)$ is an arbitrary point from $X \times R$, then

$$
\begin{aligned}
\left(\mu \times \delta_{0}\right)\left(H \times U_{2}+(x, t)\right) & =\left(\mu \times \delta_{0}\right)\left((H+x) \times\left(U_{2}+t\right)\right) \\
& =\mu(H+x) \delta_{0}\left(U_{2}+t\right)=0 .
\end{aligned}
$$

This shows that $F$ is Christensen measurable.

Now let $W_{1}=\left\{(y, t) \in R^{2}: y<t\right\}$; then $W=F^{-1}\left(W_{1}\right)$ is Christensen measurable because $W_{1}$ is closed in $R^{2}$. Q.E.D.

Let us return now to the proof of the theorem. By Lemma $6 W$ is Christensen measurable and by Lemma 3 it is midpoint convex. Hence by Lemmas 4 and 5 we deduce that $W^{\prime}=\{(x, t) \in X \times R: f(x)<t\}$ is open, or equivalently the set $\{(x, t) \in X \times R: f(x) \geqslant t\}$ is closed. This last fact implies that $f$ is upper semicontinuous on $X$ (see [1, p. 339]). Now let us consider two arbitrary points $x$ and $y$ in $X$. Then $f / e$ (the restriction of $f$ to the line $e$ going through the points $x$ and $y$ ) is upper semicontinuous; hence $f / e$ is measurable. By a theorem of Sierpinski [5] we conclude that $f / e$ is convex since $f / e$ is measurable and Jensen convex. Therefore $f$ is convex and it is well known that an upper semicontinuous convex function is continuous (see [1, p. 341]). Q.E.D.

\section{REFERENCES}

1. G. Choquet, Lectures on analysis, vol. 1, Benjamin, New York, 1969.

2. J. P. R. Christensen, On sets of Haar measure zero in abelian Polish groups, Israel J. Math. 13 (1972), 255-260.

3. Topology and Borel structure, North-Holland, Amsterdam; American Elsevier, New York, 1974.

4. L. Schwartz, Sur le théorème du graphe fermé, C. R. Acad. Sci. Paris Sér. A-B 263 (1966), $602-605$.

5. W. Sierpinski, Sur les fonctions convexes mesurables, Fund. Math. 1 (1920), 125-129.

Department of Mathematics and Statistics, University of Guelph, Guelph, Ontario, Canada Institute of Mathematics, Polish Academy of Sciences, Warsaw, Poland 